\title{
Impact of gender and dialysis adequacy on anaemia in peritoneal dialysis
}

\author{
Alicja Ryta ${ }^{1,2} \cdot$ Michal Chmielewski $^{1}$ - Alicja Debska-Slizien ${ }^{1} \cdot$ Piotr Jagodzinski $^{1}$ • \\ Malgorzata Sikorska-Wisniewska $^{1} \cdot$ Monika Lichodziejewska-Niemierko $^{1,3}[$
}

Received: 16 September 2016 / Accepted: 27 December 2016 / Published online: 5 January 2017

(C) The Author(s) 2017. This article is published with open access at Springerlink.com

\begin{abstract}
Purpose In the general population, haemoglobin $(\mathrm{Hb})$ concentration is higher in men than in women. However, target $\mathrm{Hb}$ levels in dialysis patients are set constant regardless of the patient's sex. The aim of this study was to evaluate $\mathrm{Hb}$ concentration and the use of erythropoiesis-stimulating agents (ESA) in peritoneal dialysis (PD) patients taking gender and dialysis adequacy into account.

Methods The study comprised two parts. The first was a cross-sectional analysis of $\mathrm{Hb}$ and ESA in 2180 prevalent PD patients. The second included 88 incident PD patients, followed for 36 months. During this time, the major parameters recorded at 12-month intervals included: $\mathrm{Hb}$ concentration, weekly ESA, total, renal, and peritoneal Kt/V. Erythropoietin resistance index (ERI) was calculated as the ratio between ESA dose and achieved $\mathrm{Hb}$.

Results In prevalent PD patients, $\mathrm{Hb}$ concentration was significantly lower in women, $(11.2 \pm 1.4$ vs. $11.5 \pm 1.6 \mathrm{~g} /$ $\mathrm{dl} ; p<0.001)$, despite higher doses of ESA $(2691 \pm 1821$ vs. $2344 \pm 1422 ; p=0.001)$. Hb concentrations were related to dialysis adequacy in both cohorts. However, despite significantly higher $\mathrm{Kt} / \mathrm{V}$, women were characterized by a lower $\mathrm{Hb}$ level. In incident patients, this association was present throughout the observation period, while the ESA dose in women was significantly higher at every
\end{abstract}

Monika Lichodziejewska-Niemierko

lichotek@gumed.edu.pl

1 Department of Nephrology, Transplantology and Internal Medicine, Medical University of Gdańsk, Gdańsk, Poland

2 Department of Hygiene and Epidemiology, Medical University of Gdańsk, Gdańsk, Poland

3 Department of Palliative Medicine, Medical University of Gdańsk, Gdańsk, Poland time point. In multiple regression analysis, gender was an independent determinant of ERI $(b=0.34 ; p<0.05)$.

Conclusions Despite higher dialysis adequacy, Hb concentration in women treated with PD is significantly lower, and the ability to correct it impaired, as compared to men.

Keywords Peritoneal dialysis - Gender · Dialysis adequacy $\cdot$ Anaemia $\cdot$ Erythropoiesis-stimulating agents

\section{Introduction}

Anaemia is a constant complication of end-stage renal disease (ESRD). Driven mainly by a relative deficit in erythropoietin, it has an important impact on patient's physical condition and quality of life. Vast majority of patients receive erythropoiesis-stimulating agents (ESA) to partially correct it. The target haemoglobin $(\mathrm{Hb})$ concentrations are set constant, regardless of the patient's sex [1]. This stays in contrast to the situation in the general population where $\mathrm{Hb}$ level is typically higher in men than in women. By current criteria, women are considered to be anaemic if their haemoglobin is less than 11.5 or $12.0 \mathrm{~g} / \mathrm{dl}$, while in males the threshold is set at 13.0 or $13.5 \mathrm{~g} / \mathrm{dl}$ [2]. These gender-associated disparities in $\mathrm{Hb}$ concentration are believed to be due to several factors including sex hormones, iron deficiency due to menstrual losses, and perhaps poor nutritional intake [2]. These factors are, to some extent, absent in peritoneal dialysis (PD) patients. However, it is not clear whether this would result in equal $\mathrm{Hb}$ levels and similar ESA requirements in men and women on PD.

One of the major determinants of anaemia severity and ESA responsiveness is dialysis adequacy [3]. Patients with an inadequate dialysis dose are resistant to ESA, and their 
target $\mathrm{Hb}$ is harder to reach [4]. In haemodialysis, there is an inverse relationship between the achieved $\mathrm{Kt} / \mathrm{V}$ and ESA dose, while increasing the dialysis dose is associated with an increase in haematocrit level $[5,6]$.

The primary aim of the present study was to evaluate potential associations between gender and $\mathrm{Hb}$ concentrations and ESA demand in prevalent as well as in incident PD patients. The second objective was to evaluate the potential associations between dialysis adequacy, assessed by the $\mathrm{Kt} / \mathrm{V}$, and $\mathrm{Hb}$ levels, as well as responsiveness to ESA treatment, taking gender into account.

\section{Materials and methods}

The first part of the study was performed on the basis of the national PD Registry, on 2180 prevalent PD patients. This was a cross-sectional evaluation of $\mathrm{Hb}$ concentration and ESA dose in men and women treated with PD. Obtained data also included the prevalence of co-morbidities and the total weekly Kt/V. The Polish PD Registry was established by the National Consultant in Nephrology, and it replaced PD survey in which aggregated data were gathered from all PD centres in Poland. The basic individual patient and treatment data are collected by the Registry from all the PD centres in Poland on an annual basis. The diagnosis of comorbidities was based on the information provided by the centres.

The second part was undertaken to get deeper insight into the mechanisms relating gender and dialysis adequacy to anaemia and ESA requirements of PD subjects. It was designed as a retrospective observational evaluation. All patients who initiated PD as their first renal replacement therapy (RRT) in a nephrology department of a large university-based hospital in the period between 2009 and 2013 were included. The typical dialysis prescription at baseline was four exchanges per day with a $2000 \mathrm{ml}$ low-glucose solution for patients treated with continuous ambulatory PD (CAPD), and 10,000 $\mathrm{ml}$ of low-glucose fluid overnight for subjects who started on automated PD (APD; Fresenius, Bad Homburg, Germany). All patients were followed until death, kidney transplantation, transfer to haemodialysis, or the end of the follow-up period, with a maximum followup of 36 months since the start of dialysis. During this time, the evaluated parameters were recorded at 12-month intervals. These included: $\mathrm{Hb}$ concentration, weekly ESA dosage per $\mathrm{kg}$ of body mass, total weekly $\mathrm{Kt} / \mathrm{V}$, renal Kt/V, and peritoneal $\mathrm{Kt} / \mathrm{V}$. Most patients received erythropoietin-beta as their ESA (NeoRecormon; Roche Pharma AG; Grenzach-Wyhlen, Germany). For comparisons, the weekly dose of ESA in patients taking other ESA than erythropoietin-beta was converted into erythropoietin-beta units. The erythropoietin resistance index (ERI) was calculated as the
Table 1 Baseline characteristics of the 2180 prevalent PD patients

\begin{tabular}{llll}
\hline & Men $(n=1157)$ & Women $(n=1023)$ & $p$ value \\
\hline Age & $61(48-71)$ & $61(47-72)$ & NS \\
DM (\%) & 33 & 29 & 0.03 \\
Hypertension (\%) & 83 & 75 & 0.001 \\
GN (\%) & 20 & 15 & 0.001 \\
ADPKD (\%) & 4 & 6 & 0.03 \\
Kt/V & $2.25 \pm 0.67$ & $2.47 \pm 0.74$ & 0.001 \\
Albumin (g/l) & $32.1 \pm 13.8$ & $31.4 \pm 13.9$ & $\mathrm{NS}$ \\
\hline
\end{tabular}

$D M$ diabetes mellitus, $G N$ primary glomerulonephritis, $A D P K D$ autosomal dominant polycystic kidney disease

ratio between the weekly weight-adjusted ESA dose and $\mathrm{Hb}$ concentration.

All biochemical analyses were performed using routine methods at the local laboratory. Apart from the abovementioned, they included: urea, creatinine, albumin, C-reactive protein (CRP), iron, ferritin, transferrin saturation. Diagnosis of cardiovascular disease (CVD), diabetes mellitus (DM), and arterial hypertension was made based on patients' medical records. Glomerular filtration rate (GFR) was estimated based on the MDRD equation.

Protocol of the study received approval from the Local Bioethics Committee. Results are expressed as percentages (for categorical variables), mean and standard deviation, or median and interquartile range, as appropriate. The assumption of normality was verified with the Kolmogorov-Smirnov test. A $p$ value $<0.05$ was considered to be statistically significant. Comparisons between two groups were assessed with a Student's $t$ test or a Mann-Whitney test, as appropriate. To assess correlations among the evaluated variables, Pearson's correlation coefficient $(r)$ was used. Independent associations among variables were assessed with stepwise multiple regression analysis. Statistical processing of the results was performed with the use of the statistical software STATISTICA PL v 12.0 (Statsoft, Kraków, Poland).

\section{Results}

The cross-sectional evaluation of the data from the national PD Registry was based on 2180 prevalent PD patients. The group included 1023 women and 1157 men. Their clinical characteristics are presented in Table 1 . Men were characterized by a higher prevalence of co-morbidities and a poorer dialysis adequacy, as compared to women. In the studied population, hypertensive patients had a lower $\mathrm{Hb}$ concentration, when compared to subjects with normal blood pressure $(11.2 \pm 1.6$ vs. $11.4 \pm 1.8 \mathrm{~g} / \mathrm{dl} ; p<0.05)$. Lower $\mathrm{Hb}$ in hypertensive subjects did not result from 
Table 2 Baseline characteristics of the 88 incident PD patients

\begin{tabular}{llll}
\hline & Men $(n=53)$ & Women $(n=35)$ & $p$ value \\
\hline Age (years) & $57(44-67)$ & $50(35-64)$ & NS \\
CVD $(\%)$ & 47 & 26 & 0.05 \\
DM (\%) & 34 & 31 & NS \\
Hypertension (\%) & 100 & 94 & NS \\
Body mass (kg) & $79.6 \pm 11.1$ & $62.1 \pm 12.5$ & 0.01 \\
BMI & $25.3 \pm 3.3$ & $23.3 \pm 4.5$ & 0.01 \\
Albumin (g/l) & $37.3 \pm 3.9$ & $37.06 \pm 3.6$ & NS \\
hsCRP (mg/l) & $3.85(1.60-8.20)$ & $1.40(0.70-3.80)$ & 0.01 \\
Iron (mcg/dl) & $72.9 \pm 24.5$ & $64.7 \pm 25.2$ & NS \\
Ferritin (ng/ml) & $166(83-211)$ & $140(73-332)$ & NS \\
TSAT (\%) & $27.0 \pm 11.2$ & $26.2 \pm 12.0$ & NS \\
PTH (pg/ml) & $330 \pm 241$ & $349 \pm 257$ & NS \\
Ca (mg/d) & $8.92 \pm 0.68$ & $8.85 \pm 0.76$ & NS \\
P (mg/dl) & $5.39 \pm 1.48$ & $4.71 \pm 1.28$ & 0.03 \\
RRF (ml/day) & $1423 \pm 652$ & $1205 \pm 594$ & NS \\
\hline
\end{tabular}

$C V D$ cardiovascular disease, $D M$ diabetes mellitus, $B M I$ body mass index, $h s C R P$ high-sensitivity $\mathrm{C}$-reactive protein, TSAT transferrin saturation, $P T H$ parathyroid hormone, $\mathrm{Ca}$ calcium, $\mathrm{P}$ phosphate, $R R F$ residual renal function

drugs affecting the renin-angiotensin-aldosterone system (RAAS), as $\mathrm{Hb}$ level was identical in patients with and without RAAS blockers $(11.3 \pm 1.6$ vs. $11.3 \pm 1.7 \mathrm{~g} / \mathrm{dl}$, respectively). There was a weak but significant positive association between the total $\mathrm{Kt} / \mathrm{V}$ and $\mathrm{Hb}$ level $(r=0.13$; $p<0.05)$, and a considerable negative association between the achieved $\mathrm{Kt} / \mathrm{V}$ and the required ESA dose $(r=-0.19$; $p<0.05)$. When $\mathrm{Hb}$ concentration was evaluated taking gender into account, it turned out to be significantly higher in men than in women $(11.5 \pm 1.6$ vs. $11.2 \pm 1.4 \mathrm{~g} /$ $\mathrm{dl} ; p<0.001)$. In multiple regression analysis, gender was among the independent predictors of $\mathrm{Hb}$ level, together with $\mathrm{Kt} / \mathrm{V}$, presence of diabetes mellitus and hypertension (combined $R^{2}=0.31, p<0.001$ ). Percentage of men treated with ESA was significantly lower than women $(69$ vs. $76 \% ; p<0.001$ ), and similarly, the average weekly dose of ESA was lower, as compared to women (2344 \pm 1422 vs. $2691 \pm 1821 ; p=0.001$ ).

The second part of the analysis included a total of 88 patients who started PD treatment as their initial RRT in 2009-2013. This group comprised 53 men and 35 women. The primary kidney disease was primary glomerulonephritis in 25 patients (28\%), diabetes mellitus in 15 subjects $(17 \%)$, hypertensive nephropathy in $5(6 \%)$, ADPKD in $5(6 \%)$, other and unknown in 38 patients $(43 \%)$. The prevalence of particular primary kidney diseases was comparable between men and women. Dialysis modality included CAPD in 67 patients $(76 \%)$ and APD in 21 subjects $(24 \%)$ and did not differ between genders. The

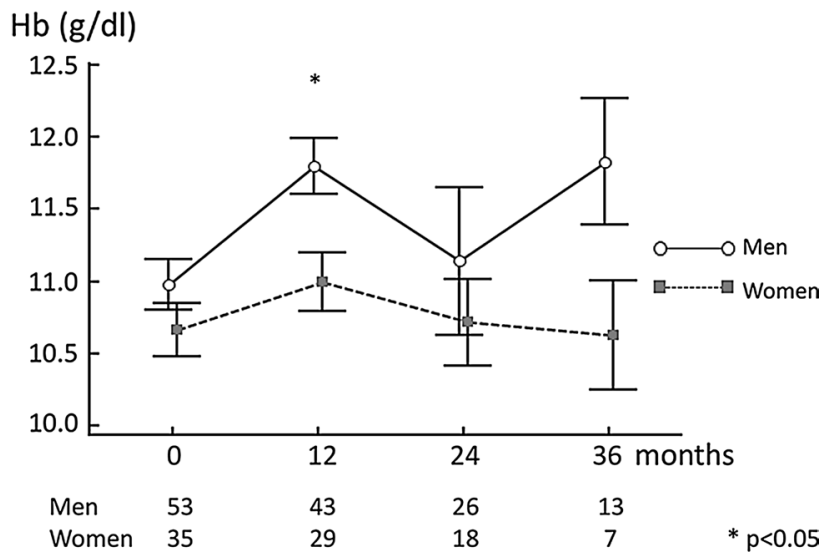

Fig. 1 Haemoglobin concentration in 88 incident PD patients

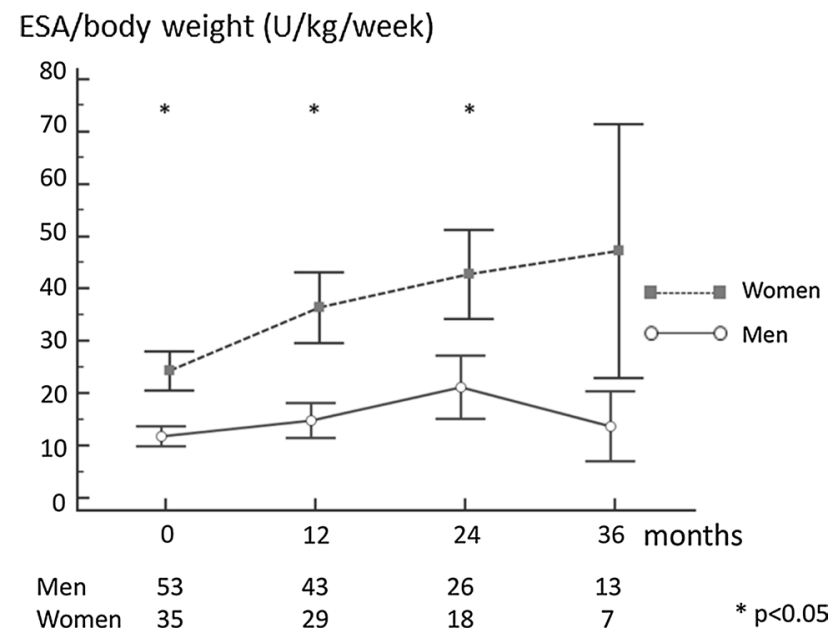

Fig. 2 Dose of ESA; ESA erythropoiesis-stimulating agents in 88 incident $P D$ patients, $B W$ body weight

baseline clinical characteristics of the studied cohort are shown in Table 2. Men were slightly older and had a higher prevalence of co-morbidities, as compared to women. At baseline, they also had an increased CRP, phosphate, and their body mass index (BMI) was significantly higher than in women. Neither of these variables turned out as significant predictors of $\mathrm{Hb}$ concentration in the multivariate analysis. The iron status was similar in both groups. Out of 53 men included in the study, ten (19\%) were on oral iron supplementation with a standard dose of $100 \mathrm{mg}$ of iron sulphate per day. In women, five $(14 \%)$ received iron at baseline $(p=$ NS). The percentage of patients prescribed iron increased during the study period and reached $47 \%$ in men and $55 \%$ in women after 3 years of follow-up ( $p=$ NS).

Men started dialysis at a higher eGFR, although this difference was not statistically significant $(9.7 \pm 3.8$ vs. $8.6 \pm 3.2 \mathrm{ml} / \mathrm{min} / 1.73 \mathrm{~m}^{2}$ ). 
The baseline $\mathrm{Hb}$ concentration was lower in women, as compared to men, similarly to the situation observed in prevalent PD patients, and in the general population; Fig. 1. It has to be underlined that, at that time point, ten women $(29 \%)$ and only three men $(6 \%)$ received ESA as a pre-dialysis treatment. The difference in $\mathrm{Hb}$ concentration persisted throughout the 36-month observation period, varying with time, being most significant after 1 year of dialysis therapy $(11.0 \pm 1.1$ vs. $11.8 \pm 1.3 \mathrm{~g} / \mathrm{dl} ; p<0.01)$. The lower $\mathrm{Hb}$ level in women was probably the major reason for high ESA doses prescribed. The mean doses remained fairly stable in men during the 3-year follow-up, while they constantly increased in women; Fig. 2. Similarly, ERI, calculated as the ratio between the ESA dose per body weight and achieved $\mathrm{Hb}$, was higher in women, as compared to men. At baseline, the median ERI equalled 2.81 (1.82$3.78)$ in women versus $2.06(1.37-2.94)$ in men $(p=0.04)$. In multiple regression analysis, following inclusion of: age, co-morbidities, CRP, albumin, iron use, and $\mathrm{Kt} / \mathrm{V}$, gender was an independent determinant of ERI $(b=0.34$; $p<0.05)$. At the end of the 36-month observation period, the median ERI was $5.66(2.85-6.16)$ in women versus 3.06 (1.46-4.88) in men, and this difference did not reach statistical significance due, mainly, to the low number of participants at that time point.

During the first year of the observation period, the total Kt/V was significantly higher in women; Fig. 3. This was predominantly due to the differences in peritoneal $\mathrm{Kt} / \mathrm{V}$ which was much higher in women, in comparison with men. This significant difference persisted through the observation period; Fig. 4. The mean peritoneal Kt/V throughout the 36 months of observation was $28 \%$ higher in women, as compared to men.

Haemoglobin concentrations turned out to be related to the dialysis adequacy, as assessed by the total Kt/V. After 12 months of dialysis treatment, the correlation coefficient for $\mathrm{Hb}$ level and $\mathrm{Kt} / \mathrm{V}$ equalled $r=0.26 ; p<0.05$. This association was similarly positive throughout the observation period, although it did not reach statistical significance at any other time point. As in prevalent patients, there was a negative relationship between the total $\mathrm{Kt} / \mathrm{V}$ and prescribed ESA dose, although not significant at any time point of the follow-up.

It has to be underlined that despite this positive association between $\mathrm{Kt} / \mathrm{V}$ and $\mathrm{Hb}$, and much higher parameters of dialysis adequacy in women, they were nevertheless more anaemic and required much higher doses of ESA, compared to men.

\section{Discussion}

The present study reveals that women treated with PD have a lower $\mathrm{Hb}$ concentration and are prescribed higher doses

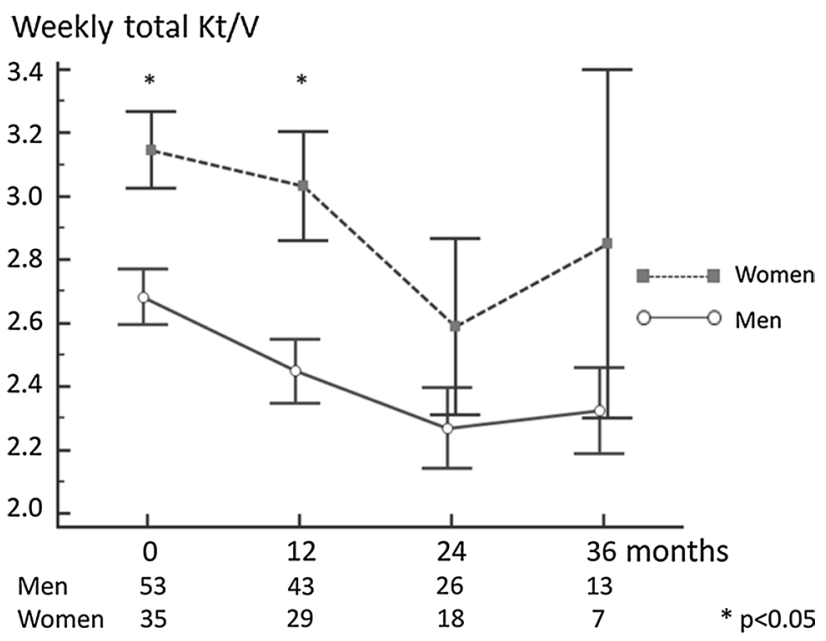

Fig. 3 Weekly total Kt/V in 88 incident PD patients

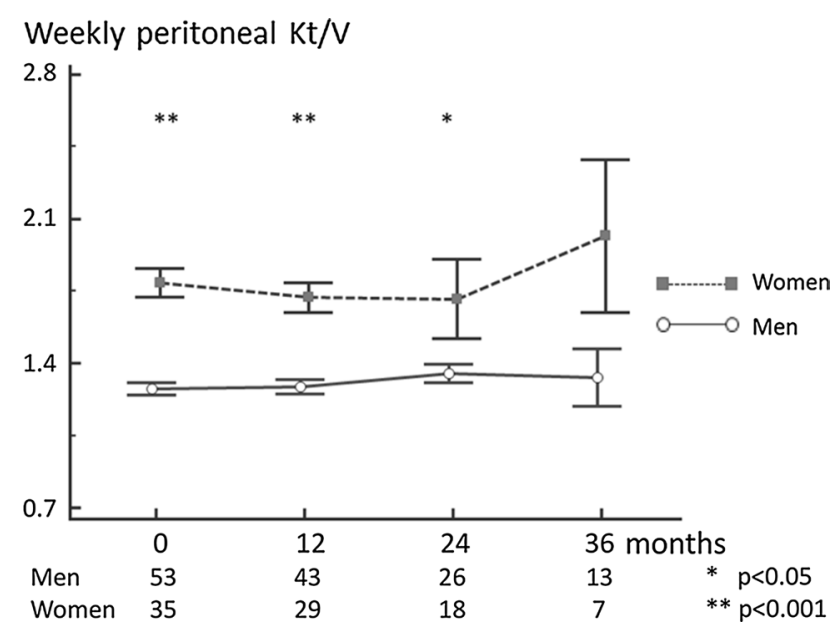

Fig. 4 Peritoneal Kt/V in 88 incident PD patients

of ESA, as compared to men. This finding was confirmed in two independent groups of prevalent and incident PD patients. Moreover, the negative impact of female gender on $\mathrm{Hb}$ level holds true despite significantly higher total $\mathrm{Kt} / \mathrm{V}$ in women, a parameter positively associated with $\mathrm{Hb}$ level.

Anaemia constitutes one of the major complications of ESRD. It impairs the patient's physical endurance and causes cognitive dysfunction deteriorating quality of life. Moreover, it negatively affects cardiac function as it leads to left ventricular hypertrophy. If severe, anaemia increases the risk of death [7]. Given its detrimental effects, it is widely acknowledged that anaemia should be treated and, partly, corrected in dialysis patients. The Kidney Disease: Improving Global Outcomes (KDIGO) guidelines on anaemia management in CKD distinguishes different thresholds for men and women to diagnose anaemia [1]. These are 
less than $13.0 \mathrm{~g} / \mathrm{dl}$ for men and below $12.0 \mathrm{~g} / \mathrm{dl}$ for women. However, indications for ESA treatment as well as the target $\mathrm{Hb}$ concentrations are set identical for both genders.

In the present evaluation, lower $\mathrm{Hb}$ levels were observed in both prevalent and incident PD women. Haemoglobin was lower despite the fact that men were characterized by an increased baseline CRP and a higher prevalence of co-morbidities, two acknowledged risk factors for anaemia severity [8]. The findings of the present study stay in accordance with previous evaluations in pre-dialysis and haemodialysis patients [4, 9]. In the general population, the reference $\mathrm{Hb}$ values are different for men and women. It is not clear whether, indeed, women require less $\mathrm{Hb}$ or whether lower concentrations result from iron deficiency in view of menstrual blood losses and, perhaps, poorer dietary intake [2]. The role of hormonal differences between genders as a contributing factor has also been proposed [2]. Similarly, in haemodialysis patients, decreased iron due to menstruations is put forward as the main contributor by some authors [10]. However, most studies have not confirmed associations between iron status and decreased $\mathrm{Hb}$ in haemodialysis women [4, 11]. According to our knowledge, no studies elucidating this issue in PD patients have been reported to date. In the present evaluation, iron status was comparable between genders in the group of incident PD patients, both at baseline and during the follow-up.

ESA responsiveness and anaemia correction in dialysis patients depends on numerous factors [12-14]. One of the major ones is dialysis adequacy [4]. Uremic toxicity in general and/or retention of some direct inhibitors of erythropoiesis results in aggravated anaemia and poor response to ESA treatment. The present study confirmed this association, as the $\mathrm{Hb}$ concentrations turned out to be related to the total $\mathrm{Kt} / \mathrm{V}$ in both studied cohorts. Moreover, in the prevalent group, $\mathrm{Kt} / \mathrm{V}$ was negatively associated with ESA dose.

In the present evaluation, women were characterized by a significantly higher $\mathrm{Kt} / \mathrm{V}$, both in the prevalent and incident group. In incident patients, this was due to significantly higher dialysis dose, as reflected by peritoneal $\mathrm{Kt} / \mathrm{V}$ throughout the observation period. Obviously, women, with their volume of urea distribution being lower than in men, are expected to achieve higher $\mathrm{Kt} / \mathrm{V}$ when prescribed the same dialysis dose [15].

However, in the present study, women, despite reaching higher adequacy parameters, presented with lower $\mathrm{Hb}$ concentrations, in comparison with men. Perhaps, it is worthy of consideration to aim for a higher $\mathrm{Kt} / \mathrm{V}$ in women in order to alleviate anaemia. On the other hand, it is well acknowledged that increasing dialysis dose above the currently recommended threshold does not lead to improved outcome [16].
Having lower $\mathrm{Hb}$ concentrations, women receive more ESA. It is well acknowledged that ESA treatment can be associated with an increase in blood pressure and with an enhanced risk of thrombotic complications [17]. Moreover, the ESA resistance index (ERI), defined as a ratio between the ESA dose and achieved $\mathrm{Hb}$, is associated with a higher risk of death in haemodialysis patients [18]. Although studies in PD patients have not shown such associations [19], the question arises as to whether it is necessary to increase ESA dose in women in order to achieve $\mathrm{Hb}$ concentrations comparable to $\mathrm{Hb}$ levels in men. It is a matter of debate and, perhaps, future studies to establish whether the increased ERI in women, observed in the present evaluation, reflects hyporesponsiveness to ESA or just physiologically lower $\mathrm{Hb}$ concentration. The issue is not only of clinical, but also of financial importance, as increasing the ESA doses in women to achieve $\mathrm{Hb}$ levels comparable to men is an expensive approach.

Our study has its limitations that ought to be underlined. First, it is an observational analysis; therefore, causal relationships cannot be assessed. Secondly, no information on the menstrual status of the studied women was obtainable. However, the comparable iron status between genders in the studied group suggests that the potential impact of menstruation on observed $\mathrm{Hb}$ levels is negligible. The Registry does not include data potentially influencing $\mathrm{Hb}$ concentration and/or dialysis adequacy, as CRP or BMI. The study has also its strengths. It has been conducted on most numerous group of PD patients in Poland so far, utilizing data from the national Registry of PD. The major findings have been verified and confirmed in incident PD patients, in a longitudinal 36-month long evaluation.

In conclusion, we have demonstrated that there is a gender-dependent difference in $\mathrm{Hb}$ concentration of patients treated with PD with women demonstrating significantly lower $\mathrm{Hb}$ despite better dialysis adequacy. This results in much higher doses of ESA prescribed. Future studies should clarify whether this approach is correct.

Acknowledgements We would like to express our gratitude to all the $\mathrm{PD}$ centres that contributed the data for the national registry of PD in Poland.

\section{Compliance with ethical standards}

Conflict of interest MLN is an employee of Fresenius NephroCare as a head of the Peritoneal Dialysis Unit at the Department of Nephrology, Transplantology and Internal Medicine. Authors received research funds, speaking honoraria (MLN and ADS), and travel grants (MLN, ADS, MC, PJ) from Baxter Healthcare and Fresenius Medical Care. $\mathrm{PJ}$ is an employee of Fresenius NephroCare PD Unit. AR and MSW declare that they have no conflict of interest.

Funding There was no funding to the study. 
Ethical approval All procedures performed in studies involving human participants were in accordance with the ethical standards of the institutional and/or national research committee and with the 1964 Helsinki Declaration and its later amendments or comparable ethical standards. The study contains retrospective observational data and Registry data. For this type of study, formal consent is not required. Protocol of the study received approval from the Local Bioethics Committee.

Open Access This article is distributed under the terms of the Creative Commons Attribution 4.0 International License (http://creativecommons.org/licenses/by/4.0/), which permits unrestricted use, distribution, and reproduction in any medium, provided you give appropriate credit to the original author(s) and the source, provide a link to the Creative Commons license, and indicate if changes were made.

\section{References}

1. KDIGO (2012) KDIGO clinical practice guideline for anemia in chronic kidney disease. Kidney Int 2(4):279-335

2. Rushton DH, Barth JH (2010) What is the evidence for gender differences in ferritin and haemoglobin? Crit Rev Oncol Hematol 73(1):1-9. doi:10.1016/j.critrevonc.2009.03.010

3. Locatelli F, Del Vecchio L (2003) Dialysis adequacy and response to erythropoietic agents: what is the evidence base? Nephrol Dial Transplant 18(Suppl 8):viii29-viii35

4. Richardson D (2002) Clinical factors influencing sensitivity and response to epoetin. Nephrol Dial Transplant 17(Suppl 1):53-59

5. Movilli E, Cancarini GC, Zani R, Camerini C, Sandrini M, Maiorca R (2001) Adequacy of dialysis reduces the doses of recombinant erythropoietin independently from the use of biocompatible membranes in haemodialysis patients. Nephrol Dial Transplant 16(1):111-114

6. Ifudu O, Feldman J, Friedman EA (1996) The intensity of hemodialysis and the response to erythropoietin in patients with endstage renal disease. N Engl J Med 334(7):420-425. doi:10.1056/ NEJM199602153340702

7. Del Vecchio L, Locatelli F (2014) Anemia in chronic kidney disease patients: treatment recommendations and emerging therapies. Expert Rev Hematol 7(4):495-506. doi:10.1586/17474086. 2014.941349

8. Stenvinkel P (2003) Anaemia and inflammation: what are the implications for the nephrologist? Nephrol Dial Transplant 18(Suppl 8):viii17-viii22

9. de Lurdes Agostinho Cabrita A, Pinho A, Malho A, Morgado E, Faisca M, Carrasqueira H, Silva AP, Neves PL (2011) Risk factors for high erythropoiesis stimulating agent resistance index in pre-dialysis chronic kidney disease patients, stages 4 and 5. Int Urol Nephrol 43(3):835-840. doi:10.1007/s11255-010-9805-9

10. Di Iorio BR, Stellato D, De Santo NG, Cirillo M, Campanian Dialysis Registry Research G (2004) Association of gender and age with erythropoietin resistance in hemodialysis patients: role of menstrual status. Blood Purif 22(5):423-427. doi:10.1159/000080234

11. Ifudu O, Uribarri J, Rajwani I, Vlacich V, Reydel K, Delosreyes G, Friedman EA (2001) Gender modulates responsiveness to recombinant erythropoietin. Am J Kidney Dis 38(3):518-522. doi:10.1053/ajkd.2001.26842

12. Wei M, Bargman JM, Oreopoulos DG (2007) Factors related to erythropoietin hypo-responsiveness in patients on chronic peritoneal dialysis. Int Urol Nephrol 39(3):935-940. doi:10.1007/ s11255-007-9226-6

13. Arikan H, Asicioglu E, Velioglu A, Nalcaci S, Birdal G, Guler D, Koc M, Tuglular S, Ozener C (2014) Determinants of hemoglobin variability in stable peritoneal dialysis patients. Int Urol Nephrol 46(7):1427-1434. doi:10.1007/s11255-014-0700-7

14. Kanbay M, Perazella MA, Kasapoglu B, Koroglu M, Covic A (2010) Erythropoiesis stimulatory agent-resistant anemia in dialysis patients: review of causes and management. Blood Purif 29(1):1-12. doi:10.1159/000245041

15. Debowska M, Paniagua R, Ventura MD, Avila-Diaz M, PradoUribe C, Mora C, Garcia-Lopez E, Qureshi AR, Lindholm B, Waniewski J (2014) Dialysis adequacy indices and body composition in male and female patients on peritoneal dialysis. Perit Dial Int 34(4):417-425. doi:10.3747/pdi.2013.00018

16. Paniagua R, Amato D, Vonesh E, Correa-Rotter R, Ramos A, Moran J, Mujais S (2002) Effects of increased peritoneal clearances on mortality rates in peritoneal dialysis: ADEMEX, a prospective, randomized, controlled trial. J Am Soc Nephrol 13(5):1307-1320

17. Blake PG, Bargman JM, Brimble KS, Davison SN, Hirsch D, McCormick BB, Suri RS, Taylor P, Zalunardo N, Tonelli M, Canadian Society of Nephrology Work Group on Adequacy of Peritoneal D (2011) Clinical practice guidelines and recommendations on peritoneal dialysis adequacy 2011. Perit Dial Int 31(2):218-239. doi:10.3747/pdi.2011.00026

18. Lopez-Gomez JM, Portoles JM, Aljama P (2008) Factors that condition the response to erythropoietin in patients on hemodialysis and their relation to mortality. Kidney Int Suppl 111:S75S81. doi:10.1038/ki.2008.523

19. Bae MN, Kim SH, Kim YO, Jin DC, Song HC, Choi EJ, Kim YL, Kim YS, Kang SW, Kim NH, Yang CW, Kim YK (2015) Association of erythropoietin-stimulating agent responsiveness with mortality in hemodialysis and peritoneal dialysis patients. PLoS ONE 10(11):e0143348. doi:10.1371/journal.pone.0143348 